\title{
Safety and efficacy of ombitasvir/paritaprevir/ritonavir/dasabuvir plus ribavirin in patients over 65 years with HCV genotype 1 cirrhosis
}

\author{
Antonio Ascione ${ }^{1}$ (1) Massimo De Luca $^{2} \cdot$ Mario Melazzini $^{3}$. Simona Montilla ${ }^{3}$ Maria Paola Trotta ${ }^{3}$. \\ Salvatore Petta ${ }^{4}$. Massimo Puoti ${ }^{5}$. Vincenzo Sangiovanni ${ }^{6}$. Vincenzo Messina ${ }^{7}$. Savino Bruno ${ }^{8}$. Antonio Izzi $^{9}$. \\ Erica Villa ${ }^{10}$. Alessio Aghemo ${ }^{11}$. Anna Linda Zignego ${ }^{12}$. Alessandra Orlandini ${ }^{13}$. Luca Fontanella ${ }^{1}$. \\ Antonio Gasbarrini ${ }^{14} \cdot$ Marco Marzioni $^{15}$. Edoardo G. Giannini ${ }^{16}$. Antonio Craxi ${ }^{4} \cdot$ For the ABACUS Study Group
}

Received: 23 January 2018 / Accepted: 22 May 2018

c) Springer-Verlag GmbH Germany, part of Springer Nature 2018

\begin{abstract}
Purpose To analyse safety and efficacy of treatment based on ombitasvir/paritaprevir/ritonavir/dasabuvir plus ribavirin in the sub-group of GT1 patients older than 65 years.

Methods We collected data extracted from the ABACUS compassionate-use nationwide Italian programme, in patients with cirrhosis due to hepatitis C virus (HCV) Genotype-1 (GT1) or 4 and at high risk of decompensation. GT1-HCV-infected patients received once-daily ombitasvir/paritaprevir, with the pharmacokinetic enhancer ritonavir $(25 / 150 / 100 \mathrm{mg})$ and twicedaily dasabuvir (250 mg) plus Ribavirin (RBV) (OBV/PTV/r + DSV + RBV) for 12 (GT1b) or 24 (GT1a) weeks. Endpoints were to evaluate safety and efficacy, the latter defined as HCV RNA negative 12 weeks after the end of treatment (SVR12). Results Patients who suffered any adverse event (AE) were 74/240 (30.8\%); 13/240 (5.4\%) discontinued the treatment. A multivariate analysis found albumin $<3.5 \mathrm{~g} / \mathrm{dL}$ (OR 2.04: 95\% CI 1.0-4.2, $p<0.05$ ) and hypertension (OR 4.6: 95\% CI 2.3-9.2, $p<0.001)$ as variables independently associated with AE occurrence. The SVR12 was 95\% (228/240). Multivariate analysis identified baseline bilirubin $<2 \mathrm{mg} / \mathrm{dL}$ (OR 4.9: 95\% CI 1.17-20.71, $p=0.029$ ) as the only variable independently associated with SVR12.

Conclusion Our findings suggest that OBV/PTV/r + DSV + RBV is safe and effective in real-life use in patients with compensated cirrhosis, HCV-GT1 infection, and age over 65.
\end{abstract}

Keywords Cirrhosis $\cdot$ Elderly $\cdot$ Ombitasvir $\cdot$ Paritaprevir $\cdot$ Dasabuvir

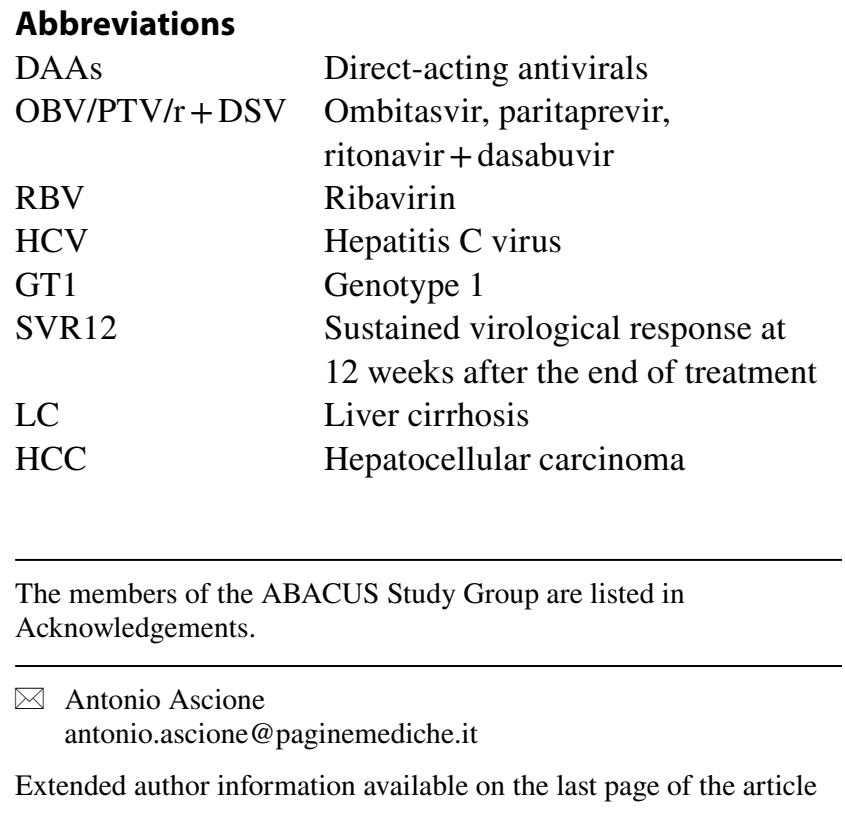

$\begin{array}{ll}\text { AIFA } & \begin{array}{l}\text { Agenzia Italiana del Farmaco } \\ \text { Associazione Italiana per lo Studio } \\ \text { del Fegato }\end{array} \\ \text { CTP } & \begin{array}{l}\text { Child-Turcotte-Pugh } \\ \text { International normalized ratio } \\ \text { INR }\end{array} \\ \text { AE } & \text { Adverse event } \\ \text { CTCAE } & \text { Common terminology criteria for } \\ \text { ITT } & \text { adverse events } \\ \text { BMI } & \text { Intention to treat } \\ \text { eGFR } & \text { Body mass index } \\ \text { MELD } & \text { Estimated glomerular filtration rate } \\ & \text { Model for end-stage liver disease }\end{array}$




\section{Introduction}

In Italy, liver cirrhosis (LC) and hepatocellular carcinoma (HCC) are the two main causes of death due to liver diseases. These two serious conditions caused, according to the last available data, 8071 deaths from LC and 10,116 from HCC [1]. Several Italian studies over the years have shown that, at least in $65 \%$ of cases, these two diseases are linked to HCV chronic infection [2-5]. In addition, the $\mathrm{HCV}$ infection is a major cause of liver transplantation in Italy [6-8].

For this reason, with the institutional endorsement of the Italian Medicines Agency (AIFA-Agenzia Italiana del Farmaco), we did the AIFA-based AISF (Associazione Italiana per lo Studio del Fegato) Compassionate Use Study (ABACUS) to provide pre-approval access to ombitasvir, paritaprevir, with the pharmacokinetic enhancer ritonavir, \pm dasabuvir + ribavirin $(\mathrm{OBV} / \mathrm{PTV} /$ $\mathrm{r} \pm \mathrm{DSV}+\mathrm{RBV}$ ) for HCV Genotype 1 (GT1) and 4 infection at high risk of decompensation. This combination, at the time of the beginning of this study, had not yet been authorized for reimbursement by the Italian National Health System, but had already shown a positive risk-benefit ratio in the registration studies [9-12]. The availability of real-world data gives the opportunity to evaluate the safety and efficacy of the OBV/PTV/r \pm DSV + RBV combination even in a sub-group of patients, such as elderly subjects, generally underrepresented in phase III trials, especially when affected by advanced fibrosis or cirrhosis. Based on these considerations, the present study reports the analysis of data from patients with cirrhosis GT1 over the age of 65 years, extracted from the group of 762 included in the already published ABACUS Study [13].

\section{Methods}

\section{Design of the study}

ABACUS was a prospective, longitudinal, observational study and not a clinical trial. The enrolment started on March 17, 2014 till May 28, 2015 when OBV/ $\mathrm{PTV} / \mathrm{r}+\mathrm{DSV}$ received the marketing approval. The study has been described in detail elsewhere [13]. One hundred and seventy-six referral centres specialized in hepatology, located in various parts of Italy and active in the AISF, participated in the ABACUS study. As ABACUS was a study to test the drug in compassionate use, a sample size was not calculated. Patients received once-daily coformulated OBV/PTV/r (25/150/100 mg) and twice-daily DSV (250 mg) for 12 (GT 1b) or 24 weeks (GT 1a) plus weight-based RBV (1000 or $1200 \mathrm{mg}$ according to the RBV label). The DAAs were provided by AbbVie (Campoverde, Italy) as the funding source. Management of side effects was not standardized by protocol. In particular, in case of anaemia, the centres were completely free to use either blood transfusion or erythropoietin or reduce the dose of RBV. Therefore, this study represents a real clinical practice in the management of antiviral therapy in Italy and provides information that usually the trials are not able to give because of the very selective enrolment criteria. This study was conducted in compliance with the International Conference on Harmonisation guidelines, the rules formulated by the Declaration of Helsinki and the applicable regulations at each study site. Local ethics approval was obtained at each site. All patients provided a written consent to participation in the study and gave permission to use their data for scientific purposes anonymously.

As with all studies where the drug is administered for compassionate use, the centres were free to organize clinical and laboratory features of the patients under their care. Investigators did laboratory assessments in their hospital lab, including international normalized ratio (INR), albumin concentration, bilirubin concentration, alanine aminotransferase (ALT) concentration, and haemoglobin concentration, at baseline, at treatment weeks 2 and 4 , every 4 weeks during treatment thereafter, and at week 12 after treatment. HCV RNA was assessed at baseline, in the fourth week of treatment, at the end of treatment, and in week 12 post-treatment. We recorded data on adverse events (AEs) throughout the study. Patient visits and follow-up, as well as laboratory testing, were done according to the sites' local standards. Laboratory and safety data were reported on an ongoing basis on a spreadsheet to the principal investigator.

Because safety was one of the primary purposes of this analysis, this aspect was checked and recorded carefully by all the centres. Any type of AE was reported in the results. AEs were graded according to Common Terminology Criteria for Adverse Events (CTCAE) Version 4.0.

The efficacy was judged by the proportion of patients who, after 12 weeks from discontinuation of therapy, showed a serum HCV RNA below the detection limit set at $<25$ IU/ $\mathrm{mL}$ (SVR12). Patients who did not become HCV RNA negative during treatment, or again became HCV RNA positive during the follow-up, were considered non-responders or relapsers.

\section{Patients}

Inclusion criteria for the enrolment in this compassionateuse prospective programme were the following: active Hepatitis C infection by GT1 or GT4 (only patients infected from the GT1 are considered in the present analysis) and compensated cirrhosis at high risk of decompensation. 
High risk of decompensation was defined as clinical features suggestive of rapidly progressive fibrosis or impending decompensation (e.g. a rapid decline in platelets, albumin concentration, or both) or with previous signs or history of decompensation, or with rapid progression from histologically or clinically proven chronic HCV infection without cirrhosis to clinically diagnosed cirrhosis. All patients were in Child-Turcotte-Pugh (CTP) Class A cirrhosis at the time of enrolment, but those with a history of decompensation were not excluded. Diagnosis of cirrhosis was made by histology from a liver biopsy, and/or Fibroscan ${ }^{\circledR}$ result $>14.6 \mathrm{kPa}$, and/or a platelet count $<100 \times 10^{9} / \mathrm{L}$, and/or the presence of oesophageal varices. Patients enrolled were treatment naïve, treatment non-responders, relapsers or intolerant to therapy based on pegylated interferon plus ribavirin. Subjects with HBV- or HIV-coinfection were not included.

\section{Statistical analysis}

As with all compassionate studies it was not appropriate to calculate a sample size. All those who met the established criteria had the right to make use of the drug before it came on the market.

Efficacy data were analysed for the intention-to-treat (ITT) population, defined as all enrolled patients who received at least one dose of study drug. All main baseline demographic and patient-related qualitative/quantitative characteristics are expressed as percentage or as mean ( \pm standard deviation) or as median (range). The only two end-point-dependent variables in the study were safety (defined as the percentage of subjects with an AE of any grade) and efficacy (defined as a proportion of patients with SVR12). Comparison between each qualitative variable and dependent variables was made using the Chi-square $\left(\chi^{2}\right)$ test or Fisher's exact test, if necessary. Comparison between each continuous variables and dependent variables was made using the $t$ test or Mann-Whitney test when indicated. In both cases, it was used a significance level defined as $p$ value less the 0.05 and $95 \%$ confidence interval (CI) of the differences. All baseline characteristics that were associated with AEs or SVR12 at univariate analysis were considered as possible independent predictors in the logistic regression model considering AEs or SVR12 as dependent variables. A multivariate analysis was performed using logistic regression with a stepwise backward method. The variables entered at the beginning of the procedure that were quantitative were categorical according to clinical criteria and were: gender, age ( $<70$ vs $\geq 70$ years); albumin (ALB) $<3.5$ vs $\geq 3.5$; total bilirubin (BilT) $<2$ vs $\geq 2$. The CTP class was not inserted because 2 of the 5 constituent parameters were absent in the initial case series (ascites, encephalopathy). Therefore, we decided to consider the quantitative parameters separately: ALB, BilT and INR.
Analysis was performed using statistical software package SPSS for windows, version 15.0. Chicago, SPSS Inc.

\section{Role of the funding source}

The funder of the study had no role in study design, data collection, data analysis, data interpretation, or writing of the report. All authors approved the manuscript and had the final responsibility for the decision to submit the data for publication.

\section{Results}

\section{Patients}

From the 762 patients, all over the age of 18 , enrolled in the ABACUS study, we extrapolated and analysed 240 patients over 65 years old (31.5\%), infected by GT1. We excluded patients infected with GT4 because the number was too small, three patients only.

The main demographic and liver disease characteristics are presented in Table 1.

In the period between enrolment and start of treatment, a change in CTP class, from A to B, was found in 16 patients $(6.7 \%)$ that were included in the analysis; they had a mean baseline (SD) age of 71.9 (5.1); male sex in $50 \%(8 / 16)$; oesophageal varices in $62.5 \%(10 / 16)$; ascites in $12.5 \%(2 / 16)$; values of serum albumin $<3.5 \mathrm{~g} / \mathrm{dL}$ in $68.8 \%$ (11/16), total bilirubin $>2 \mathrm{mg} / \mathrm{dL}$ in $50 \%$ (8/16), thrombocythemia $<100 \times 10^{9} / \mathrm{L}$ in $50 \%(8 / 16)$; one of them had a prior episode of hepatic decompensation, 12 patients (75\%) were previously treated with the antiviral therapy; 7 patients (43.8\%) had basal HCV RNA $>1 \times 10^{6} \mathrm{IU} / \mathrm{mL}$.

Seventy patients had comorbidities $(29.2 \%)$ : hypertension $45(18.8 \%)$, diabetes 16 (6.7\%), thyroid dysfunction 13 (5.4), cardiovascular disease $11(4.6 \%)$, psychiatric disorders 8 (3.3\%), and lymphoma 1 (0.4\%).

\section{Safety}

During treatment, patients who had an AE of any grade were 74/240 (30.8\%): $27 / 102$ (26.5\%) for females and 47/138 $(34.1 \%)$ for males; this difference was not statistically significant $(95 \% \mathrm{CI}-4.3$ to $18.8 \%, p=0.21)$.

As it is generally believed that the older subjects are a category more exposed to AEs, we have compared the onset of AEs in individuals younger than 70 years against those with age of 70 and over. The difference found was not statistically significant $(95 \% \mathrm{CI}-7.6$ to $15.5, p=0.49)$. Thirteen patients (5.4\%) discontinued therapy because of AEs.

Table 2 shows in detail all AEs recorded during the study. The most frequent $\mathrm{AE}$ was anaemia (12.1\%), in most cases 
Table 1 Baseline characteristics of patients $(n=240)$

\begin{tabular}{|c|c|}
\hline Male sex, $n(\%)$ & $138(57.5)$ \\
\hline Age (years), mean (SD) & $70.4(3.8)$ \\
\hline Age $>69$ years, $n(\%)$ & $125(52.1)$ \\
\hline $\mathrm{BMI}^{\mathrm{a}}\left(\mathrm{kg} / \mathrm{m}^{2}\right)$, mean $(\mathrm{SD})$ & $25.6(3.6)$ \\
\hline $\mathrm{BMI}<30\left(\mathrm{~kg} / \mathrm{m}^{2}\right), n(\%)$ & $178(74.2)$ \\
\hline Creatinine (mg/dL), mean (SD) & $0.76(0.18)$ \\
\hline $\mathrm{eGFR}^{\mathrm{b}}\left(\mathrm{mL} / \mathrm{min} / 1.73 \mathrm{~m}^{2}\right)$, mean $(\mathrm{SD})$ & $85.9(17.7)$ \\
\hline \multicolumn{2}{|l|}{ eGFR $(\mathrm{mL} / \mathrm{min}), n(\%)$} \\
\hline$\geq 90$ & $114(53.5)$ \\
\hline $60-89$ & $75(35.2)$ \\
\hline$<60$ & $24(11.3)$ \\
\hline ALT (IU/L), mean (SD) & $87.6(55.3)$ \\
\hline Total bilirubin $(\mathrm{mg} / \mathrm{dL})$, mean $(\mathrm{SD})$ & $1.12(0.59)$ \\
\hline International normalized ratio, mean (SD) & $1.15(0.26)$ \\
\hline Albumin $(\mathrm{g} / \mathrm{dL})$, mean $(\mathrm{SD})$ & $3.82(0.45)$ \\
\hline Platelets $\left(\times 10^{9} / \mathrm{L}\right)$, mean $(\mathrm{SD})$ & $113(54)$ \\
\hline Child-Turcotte-Pugh class B (\%) & $16(6.7)$ \\
\hline MELD score $\geq 10(\%)$ & $66(27.5)$ \\
\hline Fibroscan $^{\circledR}(\mathrm{kPa})$, mean $(\mathrm{SD})$ & $20.85(10.0)$ \\
\hline \multicolumn{2}{|l|}{ Esophageal varices (\%) } \\
\hline Absent & $134(55.8)$ \\
\hline Present & $74(30.8)$ \\
\hline Unknown & $32(13.3)$ \\
\hline \multicolumn{2}{|l|}{ HCV genotype (\%) } \\
\hline 1 (unknown subtype) & $5(2.1)$ \\
\hline $1 \mathrm{a}$ & $16(6.7)$ \\
\hline $1 \mathrm{~b}$ & $219(91.3)$ \\
\hline Baseline HCVRNA > $10^{6} \mathrm{IU} / \mathrm{mL}(\%)$ & $128(53.3)$ \\
\hline Treatment naïve, $n(\%)$ & $66(27.5)$ \\
\hline History of previous liver decompensation (\%) & $1(0.42)$ \\
\hline Ascites at baseline (\%) & $2(0.83)$ \\
\hline Portal thrombosis $(\%)$ & $2(0.83)$ \\
\hline Transplanted (\%) & $4(1.7)$ \\
\hline Comorbidities (any) (\%) & $70(29.2)$ \\
\hline \multicolumn{2}{|l|}{ Comorbidity $(\%)$} \\
\hline Arterial hypertension & $45(18.8)$ \\
\hline Diabetes & $16(6.7)$ \\
\hline Cardiovascular disease & $11(4.6)$ \\
\hline Thyroid dysfunction & $13(5.4)$ \\
\hline Psychiatric disorders & $8(3.3)$ \\
\hline Lymphoproliferative disorders & $1(0.4)$ \\
\hline
\end{tabular}

${ }^{a}$ BMI missing 42 (17.5\%)

${ }^{\mathrm{b}}$ Estimated glomerular filtration rate calculated by CKD-EPI

mild, and only in four cases (1.6\%) severe enough $(<8 \mathrm{~g} / \mathrm{dL})$ to require transfusions (Fig. 1a). The second most frequent $\mathrm{AE}$ was the hyperbilirubinemia (6.2\%), in five cases (2.1\%) severe ( $>5 \mathrm{mg} / \mathrm{dL}$ ), but only one interrupted the therapy for this reason (Fig. 1b). The third more frequent $\mathrm{AE}$ was asthenia (5.4\%), in all, but one, cases of mild degree. The
Table 2 Adverse events recorded during the study

\begin{tabular}{|c|c|c|c|c|}
\hline & Mild & Moderate & Severe & Total \\
\hline Anaemia & 19 & 6 & 4 & 29 \\
\hline Hyperbilirubinemia & 9 & 1 & 5 & 15 \\
\hline Asthenia & 12 & 1 & 0 & 13 \\
\hline Pruritus & 8 & 2 & 1 & 11 \\
\hline Insomnia & 6 & 1 & 0 & 7 \\
\hline Arthromyalgia & 4 & 2 & 1 & 7 \\
\hline Diarrhoea & 2 & 1 & 0 & 3 \\
\hline Pneumonia & 0 & 0 & 3 & 3 \\
\hline Dizziness & 3 & 0 & 0 & 3 \\
\hline Ascites & 2 & 0 & 0 & 2 \\
\hline Atrial fibrillation & 0 & 1 & 1 & 2 \\
\hline Glossitis & 2 & 0 & 0 & 2 \\
\hline Urinary tract infection & 1 & 0 & 1 & 2 \\
\hline Nausea & 2 & 0 & 0 & 2 \\
\hline Transient eGFR decrease & 1 & 0 & 0 & 1 \\
\hline Anxiety & 1 & 0 & 0 & 1 \\
\hline Dental abscess & 1 & 0 & 0 & 1 \\
\hline Dermatitis & 0 & 1 & 0 & 1 \\
\hline Dyspnoea & 1 & 0 & 0 & 1 \\
\hline Epigastralgia & 0 & 1 & 0 & 1 \\
\hline Epistaxis & 1 & 0 & 0 & 1 \\
\hline Encephalopathy & 1 & 0 & 0 & 1 \\
\hline Hepatocellular carcinoma & 0 & 0 & 1 & 1 \\
\hline Hepatitis flare & 0 & 1 & 0 & 1 \\
\hline Transient ischemic attack & 0 & 1 & 0 & 1 \\
\hline Arterial hypertension & 1 & 0 & 0 & 1 \\
\hline Hypokaliemia & 0 & 1 & 0 & 1 \\
\hline Arterial hypotension & 1 & 0 & 0 & 1 \\
\hline Lack of appetite & 1 & 0 & 0 & 1 \\
\hline Lymphoma & 0 & 0 & 1 & 1 \\
\hline Ocular myasthenia & 0 & 0 & 1 & 1 \\
\hline Heart palpitations & 1 & 0 & 0 & 1 \\
\hline Rash & 1 & 0 & 0 & 1 \\
\hline Herpes zoster & 1 & 0 & 0 & 1 \\
\hline Glycaemic decompensation & 0 & 0 & 1 & 1 \\
\hline Sweating & 1 & 0 & 0 & 1 \\
\hline Tachycardia & 1 & 0 & 0 & 1 \\
\hline Thoracic pain & 0 & 0 & 1 & 1 \\
\hline Cough & 1 & 0 & 0 & 1 \\
\hline Lichen planus ulcers & 1 & 0 & 0 & 1 \\
\hline Total & 86 & 20 & 21 & 127 \\
\hline
\end{tabular}

other event that occurred in at least 10 cases was pruritus (4.6\%), only in one case was it severe but treatment was not interrupted and he got SVR12.

Three patients decompensated during therapy: two due to ascites and one to encephalopathy (1.25\%). Those with ascites (both in the fourth week of treatment) stopped therapy; while the one who developed mild encephalopathy, 

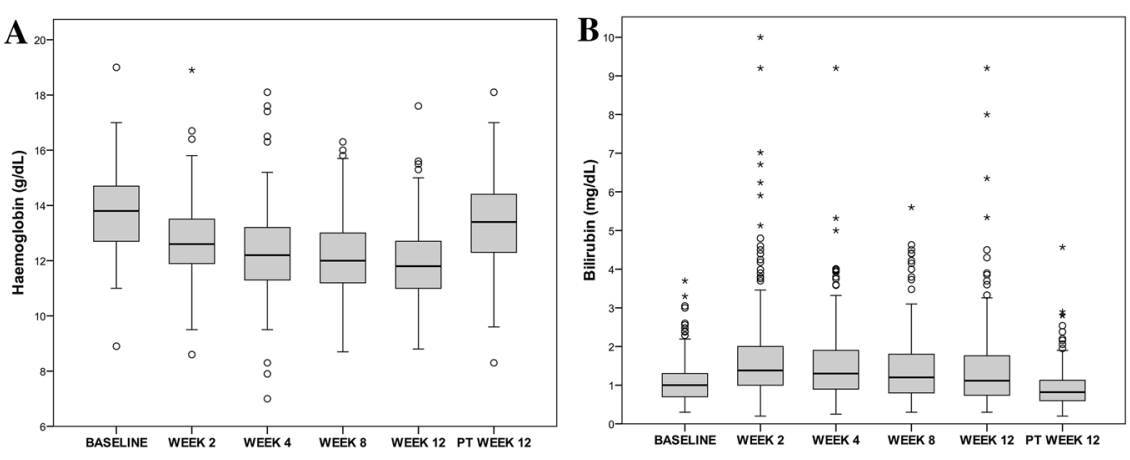

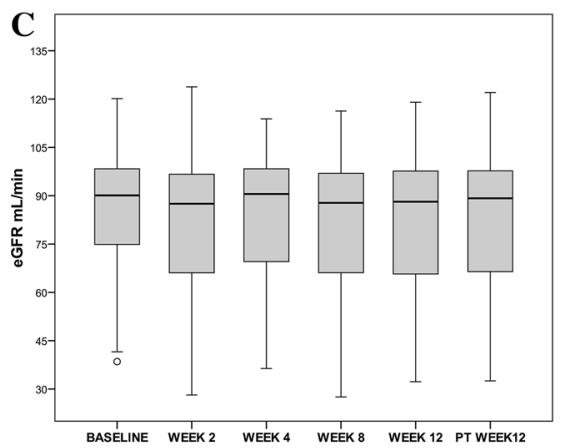

Fig. 1 Box plots for haemoglobin, bilirubin and eGFR levels during treatment and post-treatment week 12 (PT WEEK 12). a Haemoglobin at baseline vs haemoglobin at PT WEEK 12, $p=0.012$. b Bili- rubin at baseline vs bilirubin at PT WEEK 12, $p<0.001$. $\mathbf{c}$ eGFR at baseline vs eGFR at PT WEEK 12, $p=0.024$ continued the therapy. All these patients obtained the SVR12.

A group of patients needed hospitalization for: pneumonia three cases, atrial fibrillation 1 , hepatic encephalopathy 1, lymphoma 1, glycaemic decompensation 1 . In one case, a HCC was found and this had not been detected prior to the start of therapy; but may have been present as the last ultrasonography of the abdomen was performed more than 1 year before.

Two patients $(0.8 \%)$ died. The first patient died in the 12 th week of starting treatment for pneumonia and hepatopulmonary syndrome. He was a 68-year-old man, treatment naïve and had a prior episode of hepatic decompensation before being enrolled. The other patient who died was a 66-year-old male, treatment naïve, discontinued in the eighth treatment week due to the appearance of signs of lymphoma and who died for this reason in the 20th week after starting therapy.

Patients in CTP Class B had more AEs (9/16, 56.3\%) compared to those in CTP Class A $(65 / 224,29 \%)$; this difference $(27.2 \%)$ was statistically significant (95\% CI 3.3-48.6, $p=0.02$ ); however, their distribution and grade were not different from the CTP class A patients.

As for safety, in most cases there was no worsening of eGFR. Patients who at baseline had reduced eGFR $(<50 \mathrm{~mL} / \mathrm{min})$ were few $(11 / 240,4.6 \%)$, and they had no deterioration during the treatment (Fig. 1c).

A univariate analysis was conducted to verify the association between any AE and major demographic/clinical baseline variables (age, gender, BMI, previous treatments, comorbidities) and bilirubin, albumin, INR, platelet count, haemoglobin, eGFR, CTP Class and MELD score.

The only variables associated with the presence of at least one AE were: albumin less than $3.5 \mathrm{~g} / \mathrm{dL}$, CTP Class B and the presence of hypertension as comorbidity. The following baseline characteristics were found to be independently associated in multivariate logistic regression analysis: albumin
$<3.5 \mathrm{~g} / \mathrm{dL}$ (OR 2.04: 95\% CI 1.0-4.2, $p<0.05$ ) and hypertension (OR 4.6: 95\% CI 2.3-9.2, $p<0.001$ ).

In conclusion, about the correlation between AEs and therapy, only in the case of anaemia and hyperbilirubinemia, the event was connected, with reasonable certainty, to the use of ribavirin in the therapeutic schedule. As for the other AEs indicated as "severe" in Table 2, none of them was certainly ascribed by researchers to the use of study drugs.

\section{Efficacy}

The overall SVR12 rate was 95\% (228/240) among patients who received OBV/PTV/r + DSV + RBV therapy for 12 or 24 weeks. The SVR12 was similar in patients with age $<70$ years $(110 / 115,96 \%)$ compared to those $\geq 70(118 / 125$; $94 \%)$ (difference $=2 \%: 95 \%$ CI 5-7\%, $p=0.66$ ). Moreover, both in males and females, in patients with high or low BMI and in naive patients or PEG-IFN experienced there was no statistically significant difference as for SVR. As far as the viral variables are concerned, GT1a and 1b, and a viral load statistically significant differences were not found in those who achieved the SVR12: in patients with basal HCV RNA $<1 \times 10^{6}$ IU/mL (104/112, 93\%) versus those with $\mathrm{HCV}$ RNA $\geq 1 \times 10^{6} \mathrm{IU} / \mathrm{mL}(124 / 128,97 \%)$ (difference $=4 \%$ : 95\% CI -2.1 to $10.5, p=0.19$ ); in GT1b infection $(209 / 219 ; 95 \%)$ versus those with GT1a $(15 / 16$; $94 \%)($ difference $=1 \%: 95 \% \mathrm{CI}-4.6$ to $23.9 \%, p=0.76)$ and not-typeable GT1 (4/5; 80\%) (difference $=15 \%: 95 \%$ CI - 1.3-57.9\%, $p=0.59$ ) (Fig. 2).

A higher percentage of SVR12 was found in patients with a better hepatic functional reserve, CTP Class A (215/224, 96\%), compared to those with CTP Class B $(13 / 16 ; 81 \%)$ (difference $=15 \%: 95 \%$ CI $2.1-39.1$, $p=0.04)$; in patients with albumin $\geq 3.5 \mathrm{~g} / \mathrm{dL}(187 / 194$, $96 \%$ ) versus albumin $<3.5 \mathrm{~g} / \mathrm{dL}(41 / 46,89 \%)$ (difference $=7 \%: 95 \%$ CI $0.2-19.8, p=0.04)$; in patients with bilirubin $<2 \mathrm{mg} / \mathrm{dL}(210 / 219,96 \%)$ versus bilirubin $\geq 2 \mathrm{mg} /$ 


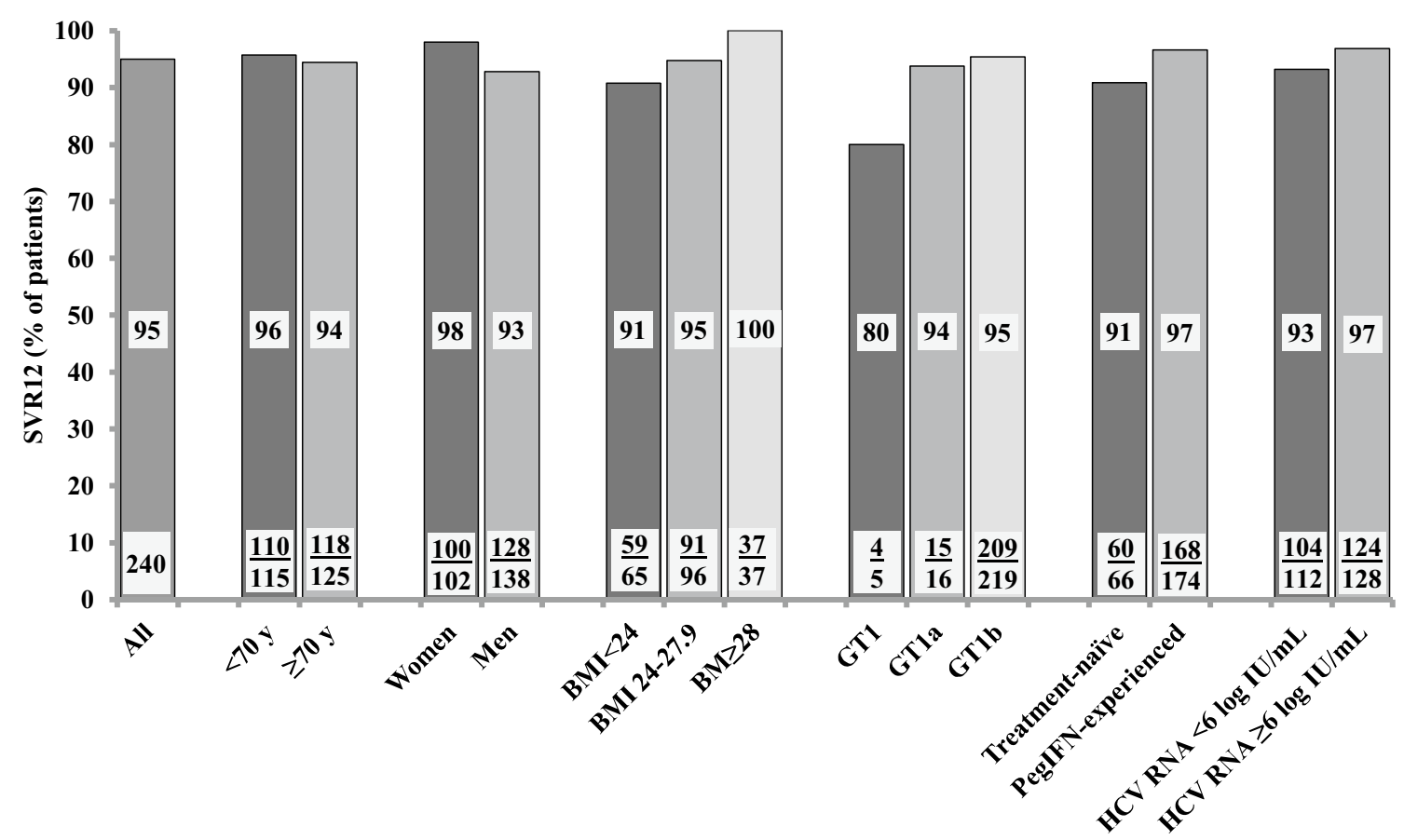

Fig. 2 Sustained virological response at post-treatment week 12 (SVR12) in all patients with cirrhosis and by age class, gender, BMI, HCV subtype, previous treatment history, HCV RNA level at base line. All comparisons were statistically not significant

$\mathrm{dL}(18 / 21,86 \%)$ (difference $=10 \%: 95 \% \mathrm{CI} 1.3-31.3$, $p=0.04)$. For model for end-stage liver disease (MELD) score, SVR12 rates were similar among patients with the MELD score $\geq 10(61 / 65,94 \%)$ in comparison with the MELD score $<10(167 / 175,95 \%)$ and this difference was not statistically significant (difference $=1 \%: 95 \% \mathrm{CI}-4.0$ to $10.5, p=0.74$ ) (Fig. 3 ).

Moreover, there was no statistical difference in patients with platelet count $\geq 100 \times 10^{9} / \mathrm{L}(122 / 127,96 \%)$ vs platelet count $<100 \times 10^{9} / \mathrm{L}(106 / 113,94 \%)$ (difference $=2 \%: 95 \%$ CI -4.5 to $7.6 \%, p=0.63)$.

Among patients who achieved SVR12, markers of liver function, including increased albumin $(p<0.001)$ and decreased bilirubin $(p<0.001)$ significantly improved in post-treatment week 12 compared with baseline, while this result did not occur for INR values ( $p=0.57$ ).

A multivariate logistic regression analysis was used to identify factors associated with SVR12; this analysis
Fig. 3 Sustained virological response at post-treatment week 12 (SVR12) by albumin, bilirubin, INR, CTP class and MELD

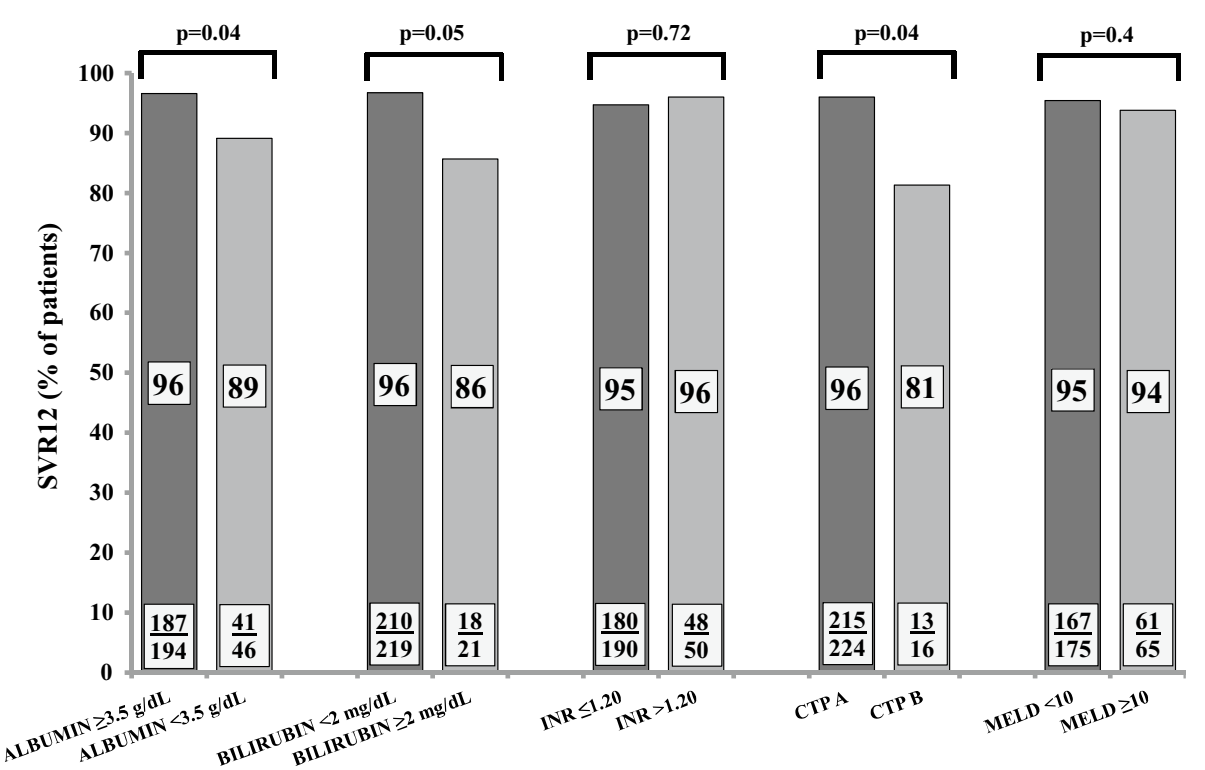


identified that baseline bilirubin $<2 \mathrm{mg} / \mathrm{dL}$ was the only variable independently associated with SVR12 (OR 4.9: 95\% CI 1.2-20.7, $p=0.02$ ).

No virological breakthrough occurred during the treatment. Twelve patients $(5 \%)$ including relapsers $(3 / 240=1.3 \%)$ did not respond to the treatment. Their median age was 70 (range 66-83); 83.3\% male, 83\% GT1b with BMI less than 24 in $54.5 \%$. This group of patients had the following baseline median (range) or occurrence number/total number (\%): $\mathrm{Hb} 12.7 \mathrm{~g} / \mathrm{dL}$ (9.9-15.1); bilirubin $1.00 \mathrm{mg} / \mathrm{dL}(0.30-3.20)$; albumin $3.8 \mathrm{~g} / \mathrm{dL}(2.5-5.1)$; INR $1.10(0.80-1.40)$; platelets count $80 \times 10^{9} / \mathrm{L}(49-169)$; ALT 73 IU/L (18-443); eGFR 90 mL/min (61-111); fibroscan $18 \mathrm{kPa}(12-48)$; HCV RNA $>1 \times 10^{6} \mathrm{IU} / \mathrm{mL}$ in $4 / 12$ (33.3\%); MELD > 10 in 4/12 (33.3\%); CTP Class B in 3/12 (25\%); oesophageal varices 6/12 (50\%); ascites $2 / 10(20 \%)$; presence of cryoglobulins $3 / 12$ (25\%); treatment naive $6 / 12$ (50\%); comorbidities 6/12 (50\%); arterial hypertension 3/12 $(25 \%)$.

\section{Discussion}

This analysis is part of a prospective compassionate-use nationwide programme including only patients infected with HCV GT1, with cirrhosis and over 65 years of age. To our knowledge, this is one of the largest real-life studies dealing with cirrhotic of old age treated with OBV/ $\mathrm{PTV} / \mathrm{r}+\mathrm{DSV}+\mathrm{RBV}$. The results, in general, surprised us, considering, above all, that the enrolled patients had at that time no therapeutic alternatives and had an advanced liver disease. Indeed, our results showed that in patients over 65 years old with compensated cirrhosis this treatment is well tolerated. It is remarkable that a population, always considered "special" or "difficult-to-treat" because of the risk of hepatic decompensation on treatment, had a relatively low percentage of any adverse events $(74 / 240=30 \%)$, an amount not different from other studies that used the same type of treatment in non-cirrhotic patients $(31.9 \%)$ [13] or using many different DAAs $(65 \%)[14,15]$. However, in a review article, specifically dedicated to the elderly infected with $\mathrm{HCV}$, the occurrence of AEs was quite high (87\%), and in those receiving ribavirin even higher (93.9\%), although the severity was in most cases mild-moderate [16], as has been reported in the present study (Table 2). The most common $\mathrm{AE}$ was anaemia, which was severe only in $1.6 \%$ of cases. Hyperbilirubinemia was the second most frequent $\mathrm{AE}$, severe in $2.1 \%$ of cases. In one case, the therapy was interrupted. Anaemia and hyperbilirubinemia (mostly unconjugated) could be related to the use of ribavirin which notoriously can cause haemolysis; but hyperbilirubinemia could also be due to the inhibition of bilirubin transport due to paritaprevir [17]; in any case, bilirubin returned to baseline during or shortly after the end of therapy. All other AEs were mild and did not hinder the normal schedule of therapy. Eight patients (3.3\%) needed hospitalization and two of them (0.8\%) died: one because of the hepatopulmonary syndrome and the second because of lymphoma. During therapy three patients developed decompensation (two ascites, one encephalopathy). However, this finding is not unexpected, bearing in mind that the patients enrolled were cirrhotic and at risk of liver decompensation. This was the condition without which the patients could not be included in this study, and for this category of patients an annual risk of $6.37 \%$ of spontaneous liver decompensation is reported [18]. The percentage of hepatic decompensation in our study is lower, but it must be said that the follow-up of our patients is 6 months only.

This study provided other relevant information concerning renal function. In fact, there was no deterioration, even in the few patients with eGFR reduced at the baseline. When we compared the AE data of this study with other similar studies of real life [19,20], we noticed that in many of them the percentages of some subjective symptoms (tiredness and headache above all) were present in significantly higher percentages than those recorded in our patients. How can this difference be explained, considering that patients in the ABACUS study were all in cirrhosis and at the same time at risk of decompensation? A possible explanation lies in the high motivation that these patients had regarding therapy and that could, therefore, be the reason why they tend to better tolerate subjective disturbances.

As for the efficacy, the percentage of SVR12 obtained in this group of patients (advanced cirrhosis, age $>65$, many non-responders/relapsers to previous antiviral treatments) was high (95\%). Analysing similar real-life studies where data for patients of the same age were reported, though mainly younger and with less hepatic impairment, the percentages of SVR12 obtained with this treatment were: 96.6\% [12]; 88.3\% [15]; 95\% [16]; 94.7\% [19]; 91.2\% [20]; 97.9-96.2 [21]. An element that should be stressed, in our study, is that a liver with reduced functional capacity at baseline significantly reduces the likelihood of SVR12. It is very important to note that this study showed that patients belonging to the CTP Class A had a significantly higher SVR12 than patients in Class B. In particular, having an albumin $<3.5 \mathrm{~g} / \mathrm{L}$ or bilirubin $>2 \mathrm{mg} / \mathrm{dL}$ significantly reduces the possibility of SVR12.

Even patients with MELD $<10$ had a better SVR12, but this difference was not found to be statistically significant. If we combine these data with the results provided by multivariate analysis, we can assume that the liver functional state is the most important predictor of virological response in this patient's category. According to the EASL recommendations, "the combination of ritonavir boosted paritaprevir and ombitasvir with or without dasabuvir is not recommended 
for patients with moderate hepatic impairment (CTP Class B) and is contraindicated in patients with severe hepatic impairment (CTP Class C)" [22]; AISF also suggests that this combined therapy is not recommended in advanced cirrhosis because there is "an increased risk of toxicity and consequent potential worsening of liver disease in patients in CTP Class B" [23].

Of course, this study has some limitations: no randomisation, no calculation of sample size, laboratory tests not centralized, and no rigid protocol for the follow-up. However, this is what happens in clinical practice every day and this is the real strength of this study: to reproduce real life exactly. The other positive feature of this study is that the centres were selected by AISF and AIFA and they were all specialized centres for the treatment of liver diseases and in patients with a viral aetiology in particular.

In conclusion, as for the SVR12, the result was certainly very high, considering the population enrolled. It is encouraging that these data differ little from that obtained in the pivotal trials of the combination OBV/PTV/r+ DSV + RBV. Also in real-life studies of this combination, the percentage of SVR12 was comparable to the results obtained from this study, and the correlation between this study and other reallife studies is a solid indicator of the data reproducibility.

Moreover, these data demonstrate that the safety of the combination OBV/PTV/r + DSV + RBV in elderly patients with compensated cirrhosis is excellent and can be used in these patients. Obviously, we need, as for all the other DAAs, long-term follow-up for a definitive demonstration that SVR12 is a reliable surrogate marker of clinical outcome and that these therapies are able to modify the natural history of elderly patients with advanced cirrhosis.

Acknowledgements The ABACUS Study Group: Giuseppe Abbati, Alfredo Alberti, Pietro Andreone, Massimo Andreoni, Paolo Angeli, Mario Angelico, Gioacchino Angarano, Debora Angrisani, Andrea Antinori, Cinzia Antonini, Ivo Avancini, Michele Barone, Raffaele Bruno, Antonio Benedetti, Veronica Bernabucci, Pier Blanc, Chiara Boarini, Nicola Boffa, Lucio Boglione, Vanni Borghi, Guglielmo Borgia, Giuseppina Brancaccio, Maurizia Brunetto, Irene Cacciola, Paolo Calabrese, Vincenza Calvaruso, Davide Campagnolo, Benedetta Canovari, Nicola Caporaso, Franco Capra, Giada Carolo, Giovanni Cassola, Francesco Castelli, Roberto Cauda, Francesca Ceccherini Silberstein, Roberto Cecere, Luchino Chessa, Alessandro Chiodera, Antonio Chirianni, Alessia Ciancio, Serena Cima, Barbara Coco, Massimo Colombo, Nicola Coppola, Giampaolo Corti, Lucio Cosco, Silvia Corradori, Raffaele Cozzolongo, Antonio Cristaudo, Elena Danieli, Antonella D'Arminio Monforte, Marco delle Monache, Paolo Del Poggio, Andrea de Luca, Chiara Dentone, Antonio Di Biagio, Alfredo Di Leo, Giovanni Di Perri, Marco Di Stefano, Giampiero D'Offizi, Francesca Donato, Emanuele Durante, Elke Erne, Stefano Fagiuoli, Katia Falasca, Alessandro Federico, Martina Felder, Carlo Ferrari, Giovanni Battista Gaeta, Roberto Ganga, Pietro Gatti, Vania Giacomet, Andrea Giacometti, Alice Gianstefani, Maria Giordani, Alessia Giorgini, Antonio Grieco, Michele Guerra, Roberto Gulminetti, Donatella Ieluzzi, Michele Imparato, Valentina Iodice, Silvia La Monica, Adriano Lazzarin, Marco Lenzi, Massimo Levrero, Myriam Lichtner, Raffaella Lionetti, Carmela Lo Guercio, Salvatore Madonna, Silvia Magnani,
Ivana Maida, Massimo Marignani, Aldo Marrone, Fabio Marsetti, Silvia Martini, Mario Masarone, Renato Maserati, Claudio Maria Mastroianni, Massimo Memoli, Barbara Menzaghi, Manuela Merli, Luca Miele, Michele Milella, Mario Mondelli, Marzia Montalbano, Monica Monti, Olivia Morelli, Filomena Morisco, Gaetano Nardone, Sergio Novara, Giovanna Onnelli, Mirella Onofrio, Simona Paganin, Luca Pani, Maria Rita Parisi, Giustino Parruti, Caterina Pasquazzi, Luisa Pasulo, Carlo Federico Perno, Marcello Persico, Guido Piai, Antonino Picciotto, Grazielle Marie Pigozzi, Sara Piovesan, Maria Chiara Piras, Massimo Pirisi, Anna Maria Piscaglia, Laura Ponti, Domenico Potenza, Cecilia Pravadelli, Mariano Quartini, Tiziana Quirino, Giovanni Raimondo, Gian Ludovico Rapaccini, Maria Rendina, Giuliano Rizzardini, Mario Rizzetto, Salvatore Rizzo, Dante Romagnoli, Antonietta Romano, Cristina Rossi, Maria Grazia Rumi, Maurizio Russello, Francesca Paolo Russo, Maria Luisa Russo, Domenico Ettore Sansonno, Teresa Antonia Santantonio, Giorgio Saracco, Anna Maria Schimizzi, Gaetano Serviddio, Filomena Simeone, Attilio Solinas, Alessandro Soria, Marco Tabone, Gloria Taliani, Giuseppe Tarantino, Pierluigi Tarquini, Marcello Tavio, Antonio Termite, Elisabetta Teti, Pierluigi Toniutto, Carlo Torti, Paolo Tundi, Giacomo Vecchiet, Gabriella Verucchi, Umberto Vespasiani Gentilucci, Maria Vinci, Vincenzo Vullo, Teresa Zolfino, and Massimo Zuin.

\section{Compliance with ethical standards}

Conflict of interest The authors declare that they have no competing interest.

\section{References}

1. Ascione A, Fontanella L, Imparato M, et al. Mortality from cirrhosis and hepatocellular carcinoma in Western Europe over the last 40 years. Liver Int. 2017;37:1193-201. https://doi.org/10.1111/ liv. 13371.

2. Amitrano L, Ascione A, Canestrini C, et al. Prevalence of antibody to hepatitis $\mathrm{C}$ virus (anti-HCV) in chronic liver diseases (CLD) in southern Italy. Ital J Gastroenterol. 1990;22:16-8.

3. Farinati F, Fagiuoli S, De Maria N, et al. Anti-HCV positive hepatocellular carcinoma in cirrhosis. Prevalence, risk factors and clinical features. J Hepatol. 1992;14:183-7.

4. Sagnelli E, Stroffolini T, Mele A, et al. The importance of HCV on the burden of chronic liver disease in Italy: a multicenter prevalence study of 9,997 cases. J Med Virol. 2005;75:522-7. https:// doi.org/10.1002/jmv.20313.

5. Ascione A, Masarone M, Tritto G, et al. Etiology of newly-diagnosed cases of chronic liver disease in Southern Italy: results of a prospective multicentric study. Ital J Med. 2014;8:176-81. https ://doi.org/10.4081/itjm.2013.409.

6. Fagiuali S, Mirante VG, Pompili M, et al. Liver transplantation: the Italian experience. Dig Liver Dis. 2002;34:640-8.

7. Belli LS, Burroughs AK, Burra P, et al. Liver transplantation for HCV cirrhosis: improved survival in recent years and increased severity of recurrent disease in female recipients: results of a long term retrospective study. Liver Transpl. 2007;13:733-40. https:// doi.org/10.1002/lt.21093.

8. Angelico M, Cillo U, Fagiuoli S, et al. Liver Match, a prospective observational cohort study on liver transplantation in Italy: study design and current practice of donor-recipient matching. Dig Liver Dis. 2011;43:155-64. https://doi.org/10.1016/j.dld.2010.11.002.

9. Poordad F, Hezode C, Trinh R, et al. ABT-450/r-ombitasvir and dasabuvir with ribavirin for hepatitis $\mathrm{C}$ with cirrhosis. N Engl $\mathrm{J}$ Med. 2014;370:1973-82. https://doi.org/10.1056/NEJMoa1402 869. 
10. Ferenci P, Bernstein D, Lalezari J, et al. ABT-450/r-ombitasvir and dasabuvir with or without ribavirin for HCV. N Engl J Med. 2014;370:1983-92. https://doi.org/10.1056/NEJMoa1402338.

11. Feld JJ, Moreno C, Trinh R, et al. Sustained virologic response of $100 \%$ in $\mathrm{HCV}$ genotype $1 \mathrm{~b}$ patients with cirrhosis receiving ombitasvir/paritaprevir/r and dasabuvir for 12 weeks. J Hepatol. 2016;64:301-7. https://doi.org/10.1016/j.jhep.2015.10.005.

12. Andreone P, Colombo MG, Enejosa JV, et al. ABT-450, ritonavir, ombitasvir, and dasabuvir achieves $97 \%$ and $100 \%$ sustained virologic response with or without ribavirin in treatment-experienced patients with HCV genotype $1 \mathrm{~b}$ infection. Gastroenterology. 2014;147:359-65. https://doi.org/10.1053/j.gastro.2014.04.045.

13. Petta S, Marzioni M, Russo P, et al. Ombitasvir, paritaprevir, and ritonavir, with or without dasabuvir, plus ribavirin for patients with hepatitis $C$ virus genotype 1 or 4 infection with cirrhosis (ABACUS): a prospective observational study. Lancet Gastroenterol Hepatol 2017;2:427-34. https://doi.org/10.1016/S2468 -1253(17)30048-1.

14. Vermehren J, Peiffer KH, Welsch C, et al. The efficacy and safety of direct acting antiviral treatment and clinical significance of drug-drug interactions in elderly patients with chronic hepatitis $\mathrm{C}$ virus infection. Aliment Pharmacol Ther. 2016;44:856-65. https ://doi.org/10.1111/apt.13769.

15. Rodríguez-Osorio I, Cid P, Morano L, et al. Real life experience with direct-acting antivirals agents against hepatitis $\mathrm{C}$ infection in elderly patients. J Clin Virol. 2017;88:58-61. https://doi. org/10.1016/j.jcv.2017.01.003.

16. Rheem J, Sundaram V, Saab S. Antiviral therapy in elderly patients with hepatitis C virus infection. Gastroenterol Hepatol. 2015;11:294-346.
17. Viekira Pak. 2017-03-23. http://www.accessdata.fda.gov/drugs atfda_docs/label/2017/206619s013s015lbl.pdf. Accessed 18 Apr 2017.

18. Alazawi W, Cunningham M, Dearden J, et al. Systematic review: outcome of compensated cirrhosis due to chronic hepatitis C infection. Aliment Pharmacol Ther. 2010;32:344-55. https://doi. org/10.1111/j.1365-2036.2010.04370.x.

19. Conti F, Brillanti S, Buonfiglioli F, et al. Safety and efficacy of direct-acting antivirals for the treatment of chronic hepatitis $\mathrm{C}$ in a real-world population aged 65 years and older. J Viral Hepat. 2017;24:454-63. https://doi.org/10.1111/jvh.12663.

20. Su F, Beste LA, Green PK, et al. Direct-acting antivirals are effective for chronic hepatitis $\mathrm{C}$ treatment in elderly patients: a real-world study of 17487 patients. Eur J Gastroenterol Hepatol. 2017;29:686-93. https://doi.org/10.1097/MEG.000000000000085 8.

21. Lawitz E, Makara M, Akarca US, et al. Efficacy and safety of ombitasvir, paritaprevir, and ritonavir in an open-label study of patients with genotype $1 \mathrm{~b}$ chronic hepatitis $\mathrm{C}$ virus infection with and without cirrhosis. Gastroenterology. 2015;149:971-80. https ://doi.org/10.1053/j.gastro.2015.07.001.

22. EASL Recommendations on Treatment of Hepatitis C 2016. European association for the study of the liver. J Hepatol. 2017;66:153-94. https://doi.org/10.1016/j.jhep.2016.09.001.

23. Documento di indirizzo dell'Associazione Italiana per lo Studio del Fegato per l'uso razionale di antivirali diretti di seconda generazione nelle categorie di pazienti affetti da epatite $\mathrm{C}$ cronica ammesse alla rimborsabilità in Italia. http://www.webaisf.org. Aggiornamento del 24 Ottobre 2017.

\section{Affiliations}

\section{Antonio Ascione ${ }^{1}$ D $\cdot$ Massimo De Luca ${ }^{2} \cdot$ Mario Melazzini $^{3} \cdot$ Simona Montilla $^{3} \cdot$ Maria Paola Trotta $^{3}$. Salvatore Petta ${ }^{4} \cdot$ Massimo Puoti $^{5}$. Vincenzo Sangiovanni ${ }^{6} \cdot$ Vincenzo Messina $^{7} \cdot$ Savino Bruno $^{8} \cdot$ Antonio Izzi $^{9}$. Erica Villa ${ }^{10}$. Alessio Aghemo ${ }^{11}$. Anna Linda Zignego ${ }^{12}$. Alessandra Orlandini ${ }^{13} \cdot$ Luca Fontanella $^{1}$. Antonio Gasbarrini ${ }^{14}$. Marco Marzioni ${ }^{15}$. Edoardo G. Giannini ${ }^{16}$. Antonio Craxì ${ }^{4}$. For the ABACUS Study Group}

1 Department of Medicine, Centre for Liver Disease, Buon Consiglio-Fatebenefratelli Hospital, Via Manzoni 220, 80123 Naples, Italy

2 Liver Unit, AORN Cardarelli, Naples, Italy

3 Italian Medicines Agency (AIFA), Rome, Italy

4 Department of Gastroenterology, DiBiMIS, University of Palermo, Palermo, Italy

5 Division of Infectious Diseases, AO Niguarda Ca' Granda Hospital, Milan, Italy

6 III U.O.C. P.O. Cotugno, AORN Ospedali dei Colli, Naples, Italy

7 Infectious Diseases Unit, AORN Caserta, Caserta, Italy

8 Humanitas University and IRCCS Clinical Institute Humanitas, Rozzano, Milan, Italy

9 Infectious Disease, Cotugno Hospital, AORN Ospedali dei Colli, Naples, Italy
10 Gastroenterology Unit, Department of Internal Medicine, AOU Policlinico of Modena, Modena, Italy

11 UO Gastroenterology and Hepatology, Foundation IRCCS Ca' Granda Hospital Maggiore Policlinico of Milan, Milan, Italy

12 Interdepartmental Centre for Systemic Manifestations of Hepatitis Viruses (MaSVE), Department of Experimental and Clinical Medicine, University of Florence, Florence, Italy

13 Unit of Infectious Diseases and Hepatology, AOU of Parma, Parma, Italy

14 Internal Medicine, Gastroenterology and Hepatology, Agostino Gemelli Hospital, Rome, Italy

15 Clinic of Gastroenterology and Hepatology, Università Politecnica delle Marche, Ancona, Italy

16 Gastroenterology Unit, Department of Internal Medicine, University of Genoa, Genoa, Italy 